\title{
Right Atrial Thrombus in a Patient With COVID-
} 19

\author{
Vittorio R. Terrigno ${ }^{1}$, Jian Liang Tan ${ }^{2}$, Devinder Singh ${ }^{2}$, Sajjad A. Sabir ${ }^{2}$ \\ 1. Internal Medicine, Cooper University Hospital, Camden, USA 2. Cardiology, Cooper University Hospital, Camden, \\ USA
}

Corresponding author: Vittorio R. Terrigno, terrigno-vittorio@cooperhealth.edu

\begin{abstract}
Coronavirus disease 2019 (COVID-19) is a worldwide pandemic. Evidence suggests a strong association between COVID-19 and pro-thrombotic states. We report our experience in managing a patient with COVID-19 complicated by a right atrial thrombus. We highlight the successful use of half-dose anticoagulation in the treatment of right atrial thrombus in a patient with COVID-19. To our knowledge, this is a first reported case of right atrial thrombus in a COVID-19 patient who was treated successfully with half-dose anticoagulation.
\end{abstract}

Categories: Cardiology, Internal Medicine

Keywords: covid 19, right atrial thrombus, thrombolytics, anticoagulation

\section{Introduction}

Coronavirus disease 2019 (COVID-19) is a dangerous infectious disease that can affect multiple organ systems. While no definitive treatment has been established, it is apparent that early diagnosis is essential for the initiation of supportive measures and treatment with the end goal of preventing rapid respiratory collapse. Hypercoagulable states as well as the many complications that can be associated with an increased state of coagulation have been strongly associated with this disease. It is important that the medical community continues to discuss and examine the appropriate preventative and therapeutic interventions to combat this specific aspect of this disease course.

\section{Case Presentation}

A 76-year-old woman with asthma and hypertension presented to the emergency room with a one-week history of dyspnea. She had an exposure to an individual who tested positive for severe acute respiratory syndrome coronavirus 2 (SARS-CoV-2). On examination, she was able to speak in full sentences. Her oxygenation saturation was $66 \%$ on room air, which improved to $94 \%$ with a non-rebreather facemask. Wheezing and diminished breath sounds were present on lung auscultation. Her respiratory status declined with an increased work of breathing within 30 minutes of her presentation, which prompted endotracheal

Received 07/10/2020 Review began 07/11/2020 Review ended 07/17/2020 Published 07/28/2020

(c) Copyright 2020

Terrigno et al. This is an open access article distributed under the terms of the Creative Commons Attribution License CC-BY 4.0., which permits unrestricted use, distribution, and reproduction in any medium, provided the original author and source are credited. intubation. Her intubation course was complicated due to significant laryngeal edema and a brief episode of pulseless ventricular tachycardia with return of spontaneous circulation after three cycles of chest compressions. She required intravenous (IV) norepinephrine to achieve a mean arterial pressure of $65 \mathrm{~mm}$ Hg. Laboratory tests demonstrated lymphopenia and abnormal biomarkers (creatinine of $1.7 \mathrm{mg} / \mathrm{dL}$, procalcitonin of $0.33 \mathrm{ng} / \mathrm{mL}$, C-reactive protein of $26 \mathrm{mg} / \mathrm{dL}$, D-dimer of $6.8 \mathrm{ug} / \mathrm{mL}$, lactate dehydrogenase of $1,132 \mathrm{U} / \mathrm{L}$, ferritin of $1,102 \mathrm{ng} / \mathrm{mL}$, and lactate of $2.2 \mathrm{mmol} / \mathrm{L})$. Her initial high-sensitivity troponin was 472 $\mathrm{ng} / \mathrm{L}$ and peaked at $606 \mathrm{mg} / \mathrm{L}$. Post-cardiac arrest transthoracic echocardiogram (TTE) revealed reduced biventricular systolic function and a mobile echo dense mass in the right atrium (RA) (Figures 1, 2 and Videos 1,2). 


\section{Cureus}

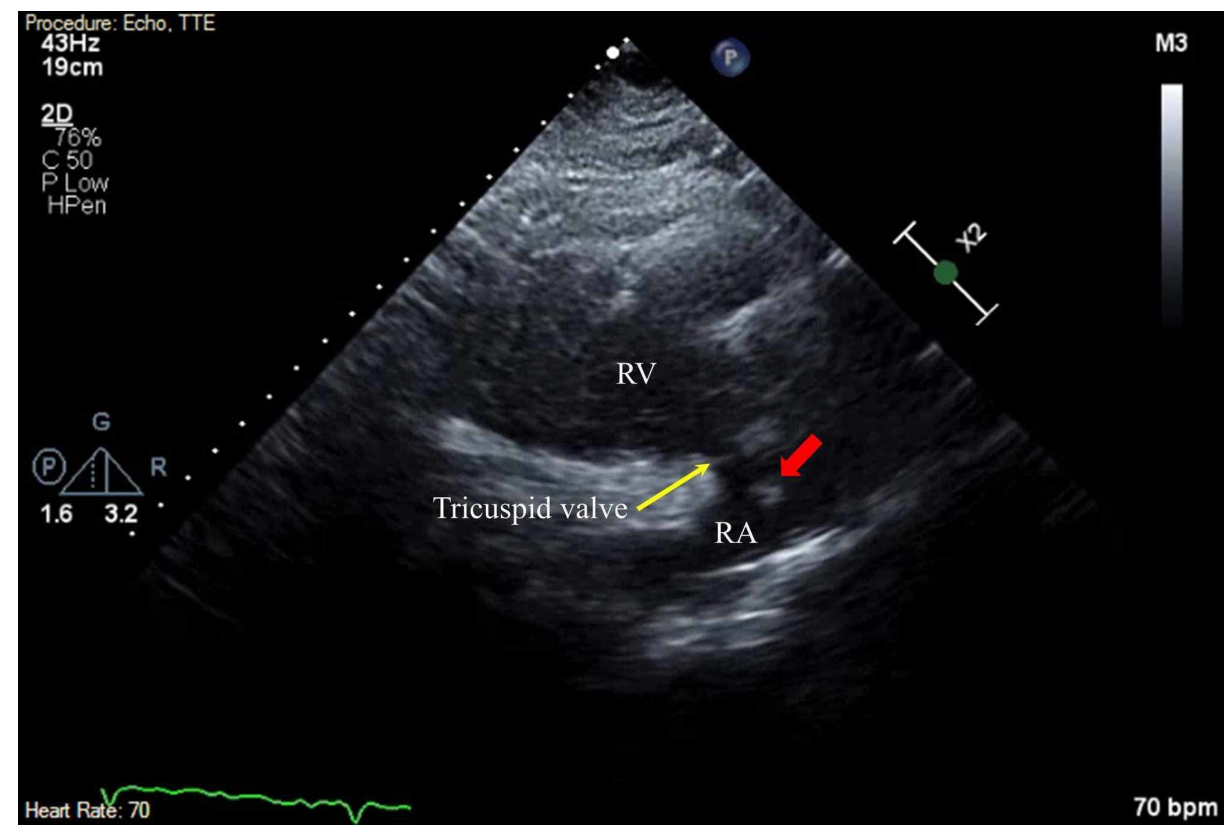

\section{FIGURE 1: Right Atrial Thrombus}

Two-dimensional TTE finding. Parasternal long-axis RV inflow tract view illustrating a mobile echo dense mass (red arrow) in the RA.

$\mathrm{RA}$, right atrium; RV, right ventricle; TTE, transthoracic echocardiogram

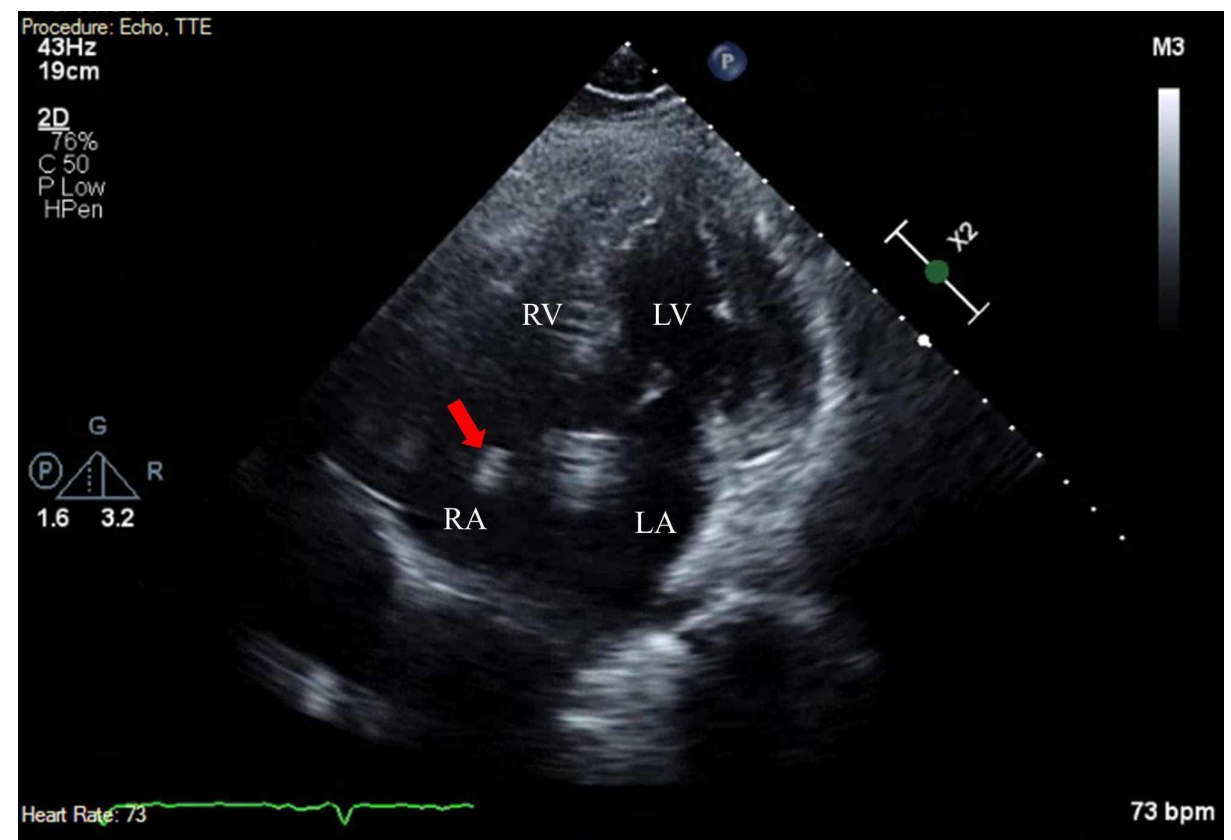

FIGURE 2: Right Atrial Thrombus: Apical Four-Chamber View

Two-dimensional TTE finding. Apical four-chamber view illustrating a mobile echo dense mass (red arrow) in the RA.

LA, left atrium; LV, left ventricle; RA, right atrium; RV, right ventricle; TTE, transthoracic echocardiography 


\section{Cureus}

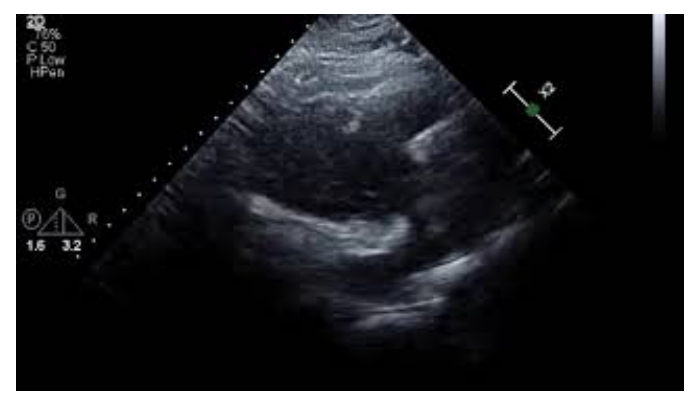

\section{VIDEO 1: Parasternal Long Axis View:- Mobile Echogenic Mass in the}

\section{Right Atrium}

Transthoracic echocardiogram in parasternal long-axis right ventricular inflow tract view showing a mobile echogenic mass in the right atrium prolapsing through the tricuspid valve inlet.

View video here: https://youtu.be/GVp9mO0lq8E

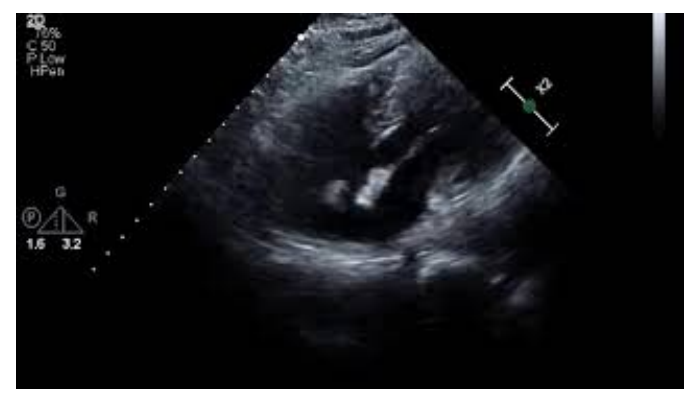

\section{VIDEO 2: Apical Five-Chamber View: Mobile Echogenic Mass in the}

\section{Right Atrium}

Transthoracic echocardiogram in apical five-chamber view showing a mobile echogenic mass in the right atrium and global hypokinesis of the left and right ventricles.

View video here: https://youtu.be/zIOTH-DV3xg

An agitated saline study did not show evidence of right to left shunting (Video 3). She was positive for SARSCoV-2 based on a real-time reverse transcription polymerase chain reaction (rRT-PCR).

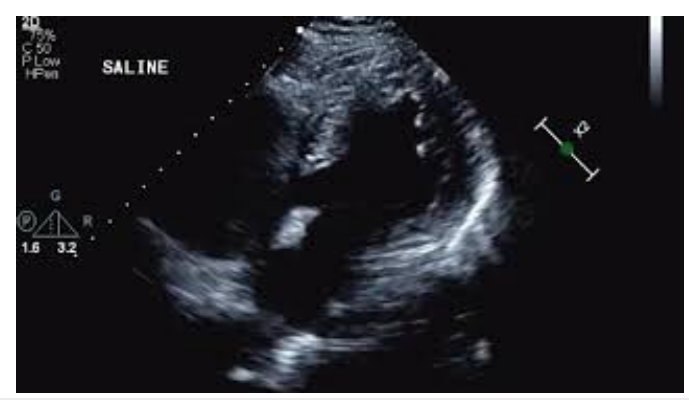

\section{VIDEO 3: Agitated Saline Study}

An agitated saline study showing no evidence of right to left shunting.

View video here: https://youtu.be/ALytlhBUPLw

Given her hemodynamic profile (increased dose of norepinephrine), a multidisciplinary team discussed various therapeutic approaches (systemic thrombolysis and surgical embolectomy) to manage the clot in the RA. A decision was made to administer $50 \mathrm{mg}$ of alteplase as a bolus over 2 minutes followed by therapeutic anticoagulation with IV heparin. This decision was made due to the patient's need for proning due to acute respiratory distress syndrome and also her need for close monitoring for potential bleeding. Due to these variables, which many patients with COVID-19 face, half-dose alteplase therapy was given instead of the full dose. A repeat TTE was performed on hospital day 4, which demonstrated resolution of the clot (Video 4). 


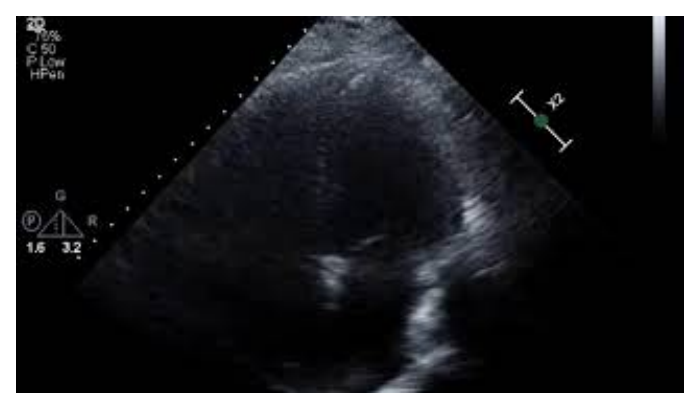

\section{VIDEO 4: TTE demonstrating RA Clot Resolution}

TTE in apical four-chamber view at day 4 post-tissue plasminogen activator administration and while on continuous heparin infusion showed absence of RA clot and improvement in biventricular systolic function.

TTE, transthoracic echocardiography; RA, right atrium

View video here: https://youtu.be//n2TRjHOFp4

She was eventually taken off of IV norepinephrine on hospital day 9 and showed some clinical signs of improvement. However, despite receiving hydroxychloroquine, azithromycin, and lopinavir/ritonavir, her respiratory status once again worsened. Her family decided to proceed with a compassionate extubation due to worsening of her COVID-19 illness and she expired on hospital day 13. Due to hospital protocol during the COVID-19 pandemic and the family's wish, autopsy was not performed.

\section{Discussion}

In a retrospective single center study of 81 COVID-19 ICU patients from China, 25\% (20/81) of the patients developed venous thromboembolism (VTE). These patients had an elevated D-dimer, similar to our patient. None of the patients had received VTE prophylaxis [1]. In another three-center retrospective study of 184 COVID-19 ICU patients from the Netherlands, the authors reported 31\% incidence of thrombotic complications. All patients received standard thromboprophylaxis. Of note, the diagnostic testing was only carried out in patients with a suspicion of thrombotic complications [2]. Therefore, the true incidence is likely higher.

Our case supports the need to think of initiating therapeutic anticoagulation early in the course of critically ill patients with COVID-19. In a publication by an international collaborative of clinicians and investigators, a majority of the panel members recommended prophylactic dose anticoagulation, but a minority supported considering even intermediate to therapeutic dose anticoagulation [3]. In addition to this, our case also highlights the use of half-dose anticoagulation therapy to treat a right atrial thrombus. Patients with COVID-19 may need prone positioning, which makes monitoring for bleeding difficult and frequent neurological checks difficult. In addition to this, patients with other bleeding risks may not be able to receive full-dose anticoagulation. To our knowledge, this is the first reported case of right atrial thrombus in a COVID-19 patient that resolved with a half dose of thrombolytic therapy.

\section{Conclusions}

Our case report highlights the continued need for the consideration of early anticoagulation in patients with COVID-19. Numerous studies have shown a direct correlation between hypercoagulable states and COVID19 infection. We highlight the ability to treat a right atrial thrombus in a patient with COVID-19 with halfdose anticoagulation. This may be beneficial in patients with COVID-19 who require prone positioning and frequent neurological checks as well as for COVID-19 patients who are at higher risk when receiving thrombolytic therapy.

\section{Additional Information \\ Disclosures}

Human subjects: Consent was obtained by all participants in this study. IRB Cooper University Hospital issued approval $\mathrm{n} / \mathrm{a}$. The patient in our case report is deceased and we were unable to reach her next of kin for consent. We contacted our IRB Department requesting an approval letter, but it was deemed that our case report does not meet the definition of a human subject per 45 CFR $46.102(f)$ and would not fall under the IRB purview. We took great care to ensure that we did not include any identifiable patient health information in our case report. . Conflicts of interest: In compliance with the ICMJE uniform disclosure form, all authors declare the following: Payment/services info: All authors have declared that no financial support was received from any organization for the submitted work. Financial relationships: All authors have declared that they have no financial relationships at present or within the previous three years with any organizations that might have an interest in the submitted work. Other relationships: All authors have 


\section{Cureus}

declared that there are no other relationships or activities that could appear to have influenced the submitted work.

\section{References}

1. Cui S, Chen S, Li X, Liu S, Wang F: Prevalence of venous thromboembolism in patients with severe novel coronavirus pneumonia. J Thromb Haemost. 2020, 18:1421-1424. 10.1111/jth.14830

2. lok FA, Kruip MJHA, van der Meer NJM, et al.: Incidence of thrombotic complications in critically ill ICU patients with COVID-19. Thromb Res. 2020, 191:145-147. 10.1016/j.thromres.2020.04.013

3. Bikdeli B, Madhavan MV, Jimenez D, et al.: COVID-19 and thrombotic or thromboembolic disease: implications for prevention, antithrombotic therapy, and follow-up: JACC State-of-the-Art Review. J Am Coll Cardiol. 2020, 75:2950-2973. 10.1016/j.jacc.2020.04.031 